\title{
Artisanat antique dans l'aire vésuvienne : le cas de la pierre à Pompéi et Herculanum
}

Rapport d'activité 2013

Guilhem Chapelin, Ghislain Vincent et Marina Covolan

\author{
CpenEdition \\ Journals \\ Édition électronique \\ URL : http://journals.openedition.org/cefr/1166 \\ DOI : $10.4000 /$ cefr. 1166 \\ ISSN : 2282-5703 \\ Éditeur \\ École française de Rome
}

Référence électronique

Guilhem Chapelin, Ghislain Vincent et Marina Covolan, «Artisanat antique dans l'aire vésuvienne : le cas de la pierre à Pompéi et Herculanum », Chronique des activités archéologiques de l'École française de Rome [En ligne], Les cités vésuviennes, mis en ligne le 14 avril 2014, consulté le 11 septembre 2019 URL : http://journals.openedition.org/cefr/1166 ; DOI : 10.4000/cefr.1166

Ce document a été généré automatiquement le 11 septembre 2019.

(c) École française de Rome 


\title{
Artisanat antique dans l'aire vésuvienne : le cas de la pierre à Pompéi et Herculanum
}

Rapport d'activité 2013

\author{
Guilhem Chapelin, Ghislain Vincent et Marina Covolan
}

\section{NOTE DE L'AUTEUR}

Nous tenons à remercier ici toutes les institutions et les personnes qui ont rendu possible le démarrage de cette étude : l'Inrap, le Centre Jean Bérard, l'École française de Rome, le CNRS, la Soprintendenza speciale per i Beni archeologici di Napoli e Pompei et le Ministère des Affaires étrangères.

Cette étude est née dans un cadre pluri-institutionnel, l'Inrap d'une part et le Centre Jean Bérard (USR 3133 CNRS-EFR) de l'autre. Elle s'insère dans le programme « artisanat » du Centre Jean Bérard. En 2012 et 2013 trois campagnes ont eu lieu en collaboration avec la Soprintendenza speciale per i Beni archeologici di Napoli e Pompei. Le projet scientifique en lui-même porte sur l'artisanat de la pierre. Cette recherche vise à parfaire notre connaissance des techniques de travail, de l'organisation professionnelle des tailleurs de pierre et de leur place dans l'économie de l'Antiquité. La recherche porte sur des éléments porteurs de petits modules en tuf volcanique gris dit «de Nocera $»^{1}$ qui se rencontrent dans de nombreux bâtiments de Pompéi et d'Herculanum (domus, lieux publics, ateliers, etc. $)^{2}$.

2 L'usage du tuf gris à Pompéi semble avoir pu commencer entre le début du III

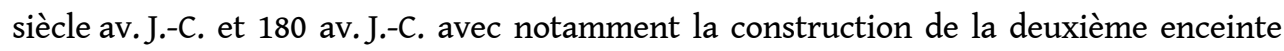
samnite ${ }^{3}$. Ainsi, il se peut que la production des colonnettes et des piliers qui nous intéressent se soit faite sans interruption depuis cette période jusqu'à la période augustéenne à partir de laquelle A. Maiuri semble limiter son usage à quelques rares structures secondaires constituées en matériaux de remploi ${ }^{4}$. Les blocs de pierre taillée 
qui sont le point de départ de cette étude constituaient de petits éléments porteurs à savoir des colonnes et des piliers à demi-colonnes engagées avoisinant les $2 \mathrm{~m}$ de hauteur. Il s'agit d'une production spécifique liée à la construction des seconds niveaux des maisons qui permettait de créer des loggias largement ouvertes recevant éventuellement des menuiseries. On retrouve aujourd'hui la plupart de ces blocs à terre, dans les maisons, dans certaines rues ou stockés dans les dépôts du site mais il est difficile de reconstituer des séries. L'idée générale sous-tendue par ce travail est d'essayer, par la caractérisation de chaînes opératoires et la mise en place de typologies d'éléments architecturaux, de définir des ensembles cohérents révélant la signature d'un ou au contraire de plusieurs ateliers œuvrant sur ces sites et fournissant des produits similaires aux commanditaires de travaux de construction. Tous les blocs dispersés dans Pompéi que nous avons à étudié présentent des caractéristiques communes: ils peuvent marquer la présence d'une fabrication rapide et unitaire dont la production se diffuse largement dans la ville et peut-être même, comme nous le pressentons, sur un territoire beaucoup plus vaste car des pièces architecturales assez semblables ont pu être observées à Herculanum à une quinzaine de kilomètres de là.

\section{Organisation et méthodologie}

Fig. 1 - Exemple de blocs couverts par mousses et lichens qui nécessitent un important nettoyage pour étude
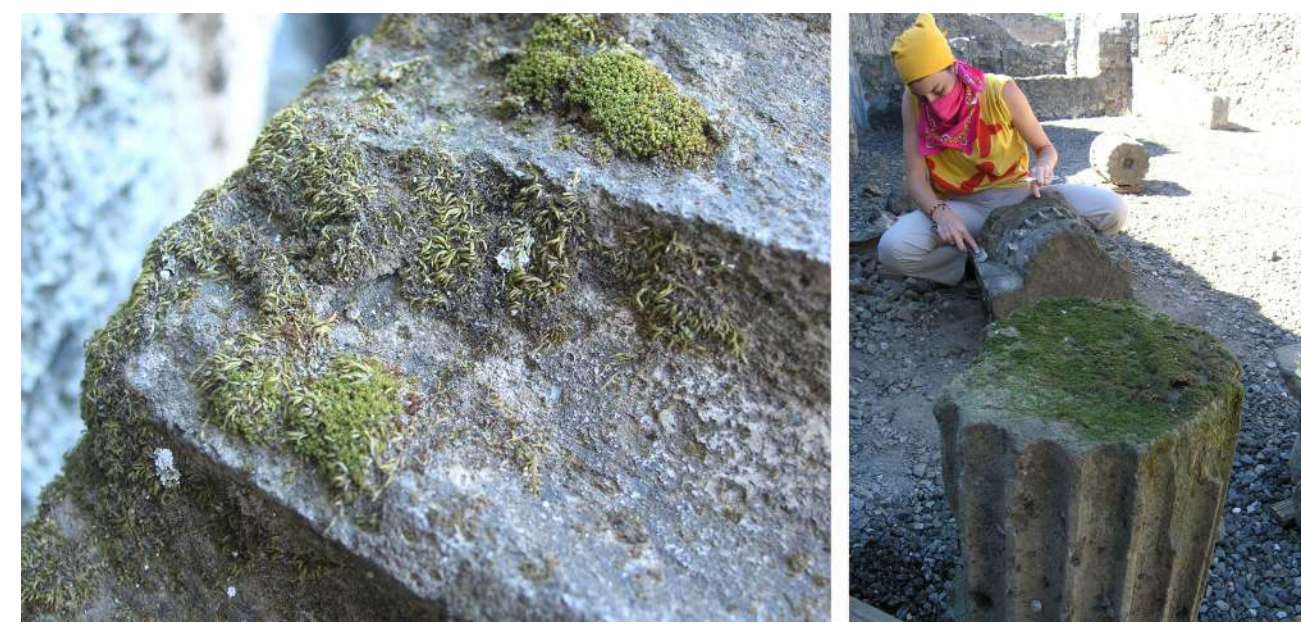

G. Chapelin, M. Covolan.

D’un point de vue général, les recherches comportent un volet de prospections sur le terrain afin de localiser les blocs. Ce travail permet, en outre, de produire une cartographie de leur localisation (fig. 2). 
Fig. 2 - Pompéi. Situation des blocs recensés en novembre 2013.

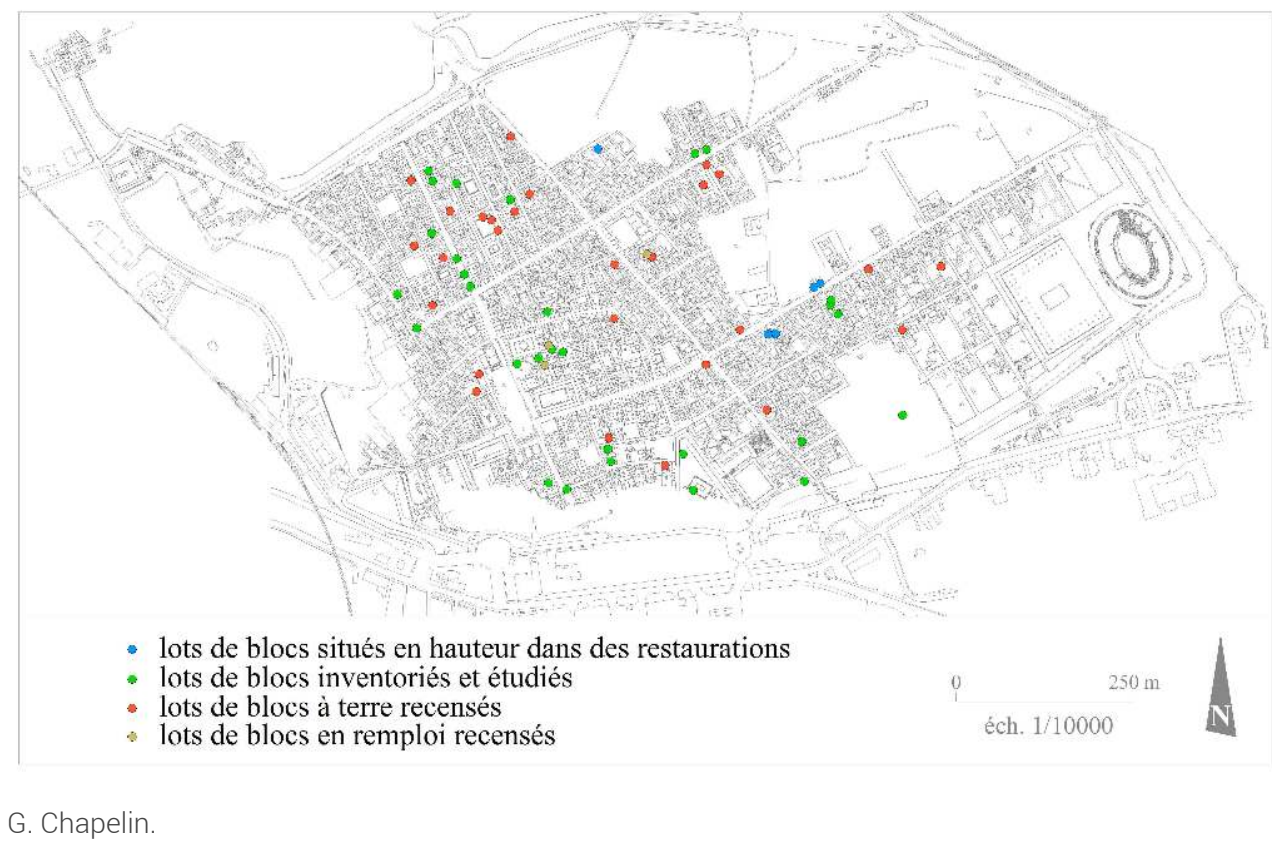

Le travail le plus long concerne le nettoyage des pièces afin de retrouver l'épiderme originel (fig. 1). Nous réalisons alors un inventaire et une étude de chacun des blocs. Sont recensés et décrits les modules, les mortaises, les feuillures et quand elles sont observables toutes les traces techniques laissées par le travail de l'artisan comme par exemple celles laissées par un tour, un ciseau, un marteau taillant ou un foret. En parallèle, une couverture photo est produite pour chaque bloc (fig. 3). Enfin, des dessins techniques sont effectués pour certains éléments, notamment des profils de moulures de bases (fig. 4).

Fig. 3 - Exemple de couverture photo générale du bloc 11.

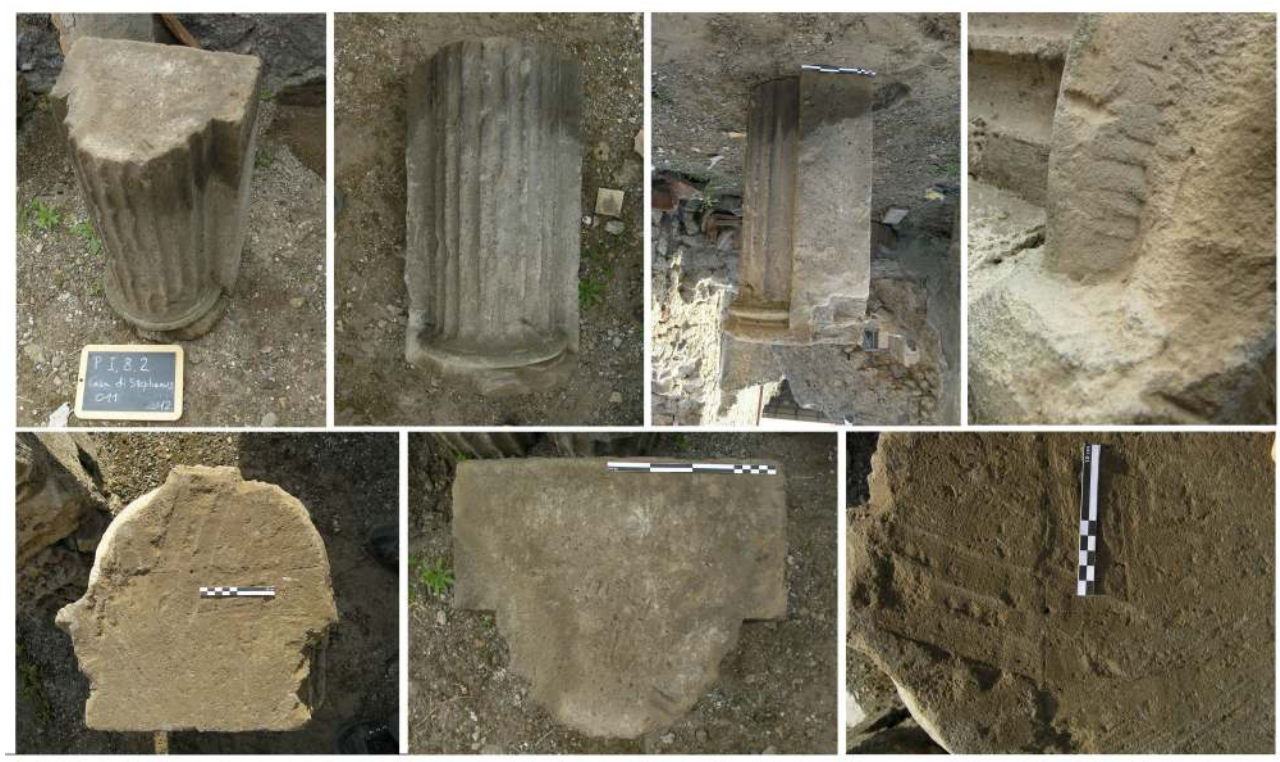

G. Chapelin, G. Vincent. 
Fig. 4 - Profils comparés de moulures de base de colonnes libres.

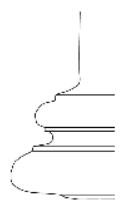

025

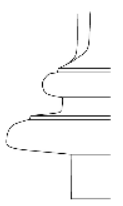

056

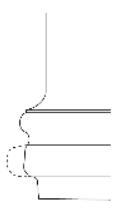

090

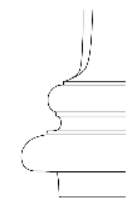

028

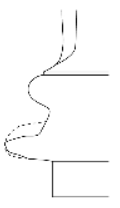

057

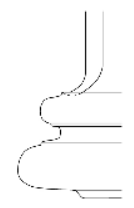

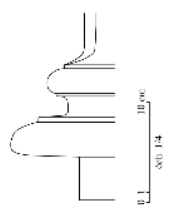

055
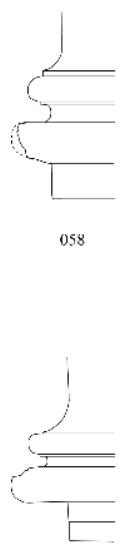

107

G. Chapelin, G. Vincent.

5 L'un des apports de la prospection 2013 a été le repérage de nombreux blocs fragmentés et remployés dans les murs de maisons ${ }^{5}$ et même dans des bordures de trottoir ${ }^{6}$ (fig. 5). 
Fig. 5 - Trottoir devant VII, 9, 39 où un tronçon de pilier a demi-colonne engagée a été remployé.

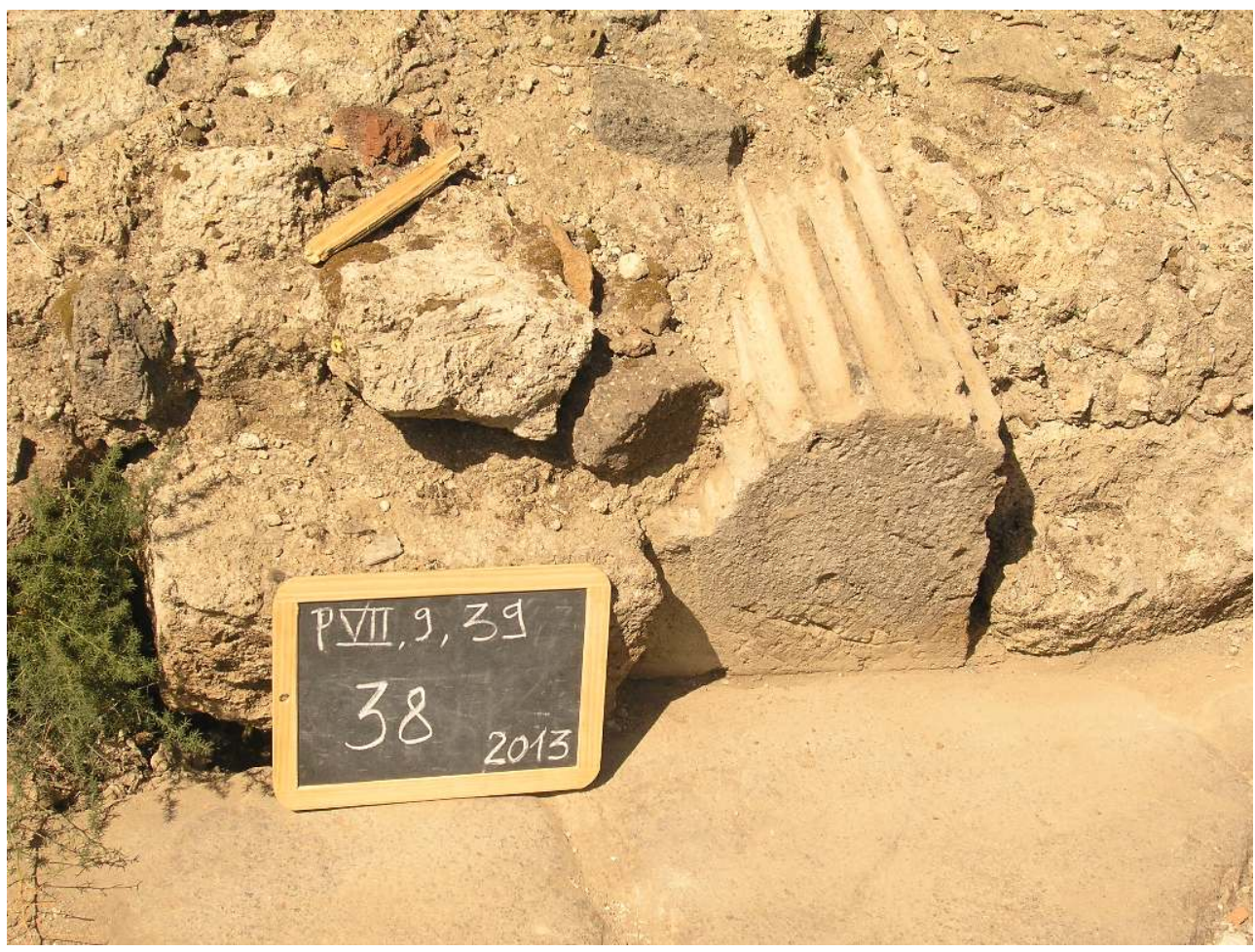

G. Chapelin, G. Vincent.

6 Le cas des blocs en remploi est particulier. D'un côté l'étude de toutes leurs faces est impossible, mais d'un autre côté leur lien avec des structures en place nous permet d'espérer que les données de fouille, si elles existent, nous apporteront une datation de la construction ou de la réfection de ces murs et donc un terminus ante quem pour la fabrication des blocs.

\section{Typologie, style et technique de taille}

7 L'étude a permis de commencer, au moins provisoirement, un classement typologique des éléments architecturaux répertoriés. Ces éléments porteurs peuvent se classer en deux grands ensembles : des piliers à demi-colonne engagée et des colonnes libres (fig. 6). Se distinguent ensuite pour chacun de ces éléments des sous-ensembles : ceux portant des cannelures et ceux restés lisses, ceux portant des chapiteaux cubiques et ceux portant des chapiteaux décorés doriques, ioniques ou corinthiens. 
Fig. 6 - Exemple de piliers à demi-colonnes engagées à gauche et de colonnes libres à droite.
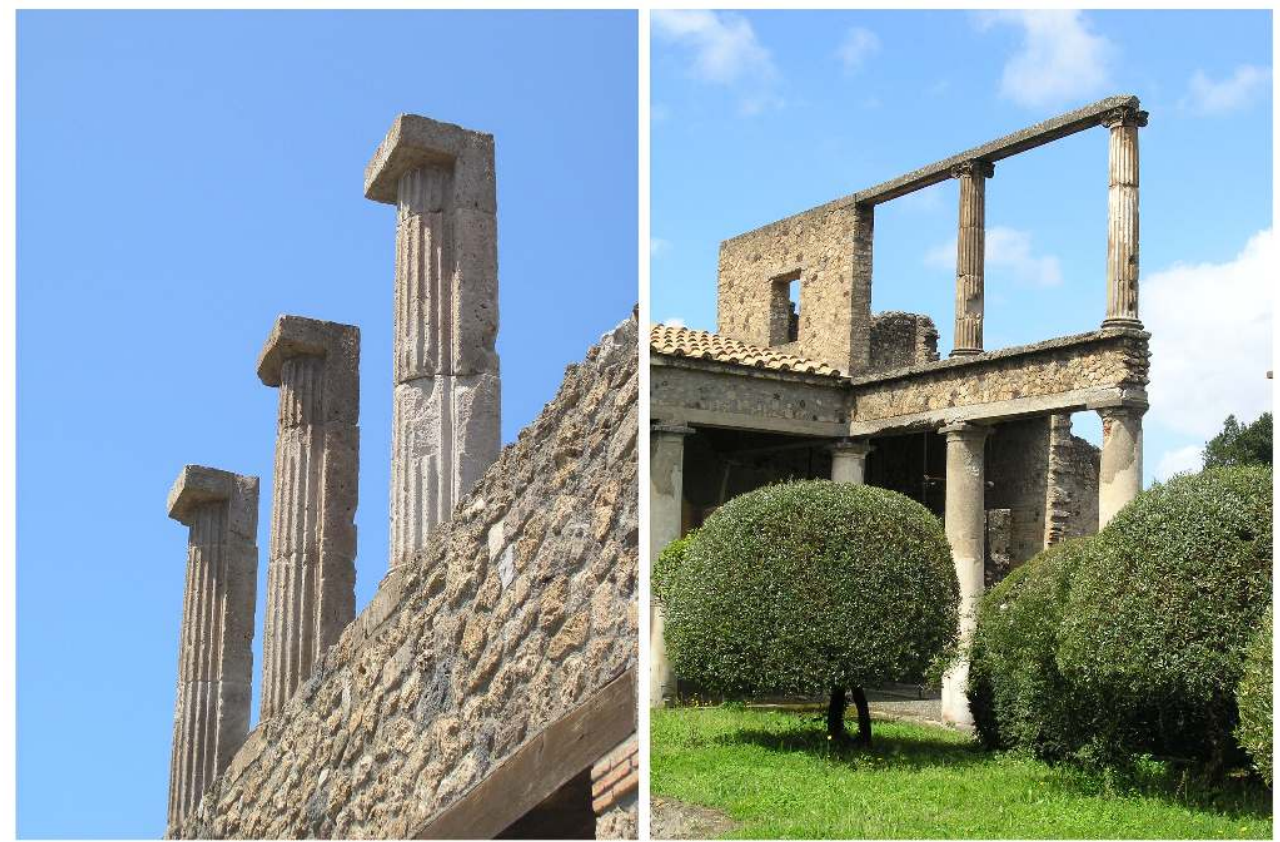

G. Chapelin, G. Vincent.

Ceci appelle quelques réflexions relatives aux chapiteaux cubiques, très nombreux sur le site de Pompéi. Ces derniers ont déjà fait l'objet de plusieurs études ${ }^{7}$. Il s'agit d'une forme particulière de finition, en parallélépipède simple, mais dont le travail est quand même totalement abouti comme l'indique l'abrasion totale des faces. Même si quelques exemplaires peuvent avoir été des pièces non finalisées, la plupart des blocs que nous avons rencontrés sont totalement achevés (voir à titre, le bloc 27 de notre inventaire, fig. 7). Les exemples les plus parfaits qui présentent cette caractéristique se retrouvent sans aucun doute dans les éléments de pilier à demi-colonne engagée, dont les chapiteaux sont, en l'état actuel des recherches, toujours cubiques et finalisés comme tels. Par conséquent, même s'il s'agit à l'origine d'une forme qui découle d'un non aboutissement de la taille pour des raisons purement techniques ou financières, il faut bien admettre que cette caractéristique devient prépondérante sur le site d'un point de vue stylistique ${ }^{8}$. 
Fig. 7 - Exemple de chapiteau cubique terminé et abrasé jusqu'au contact des cannelures : chapiteau 27.

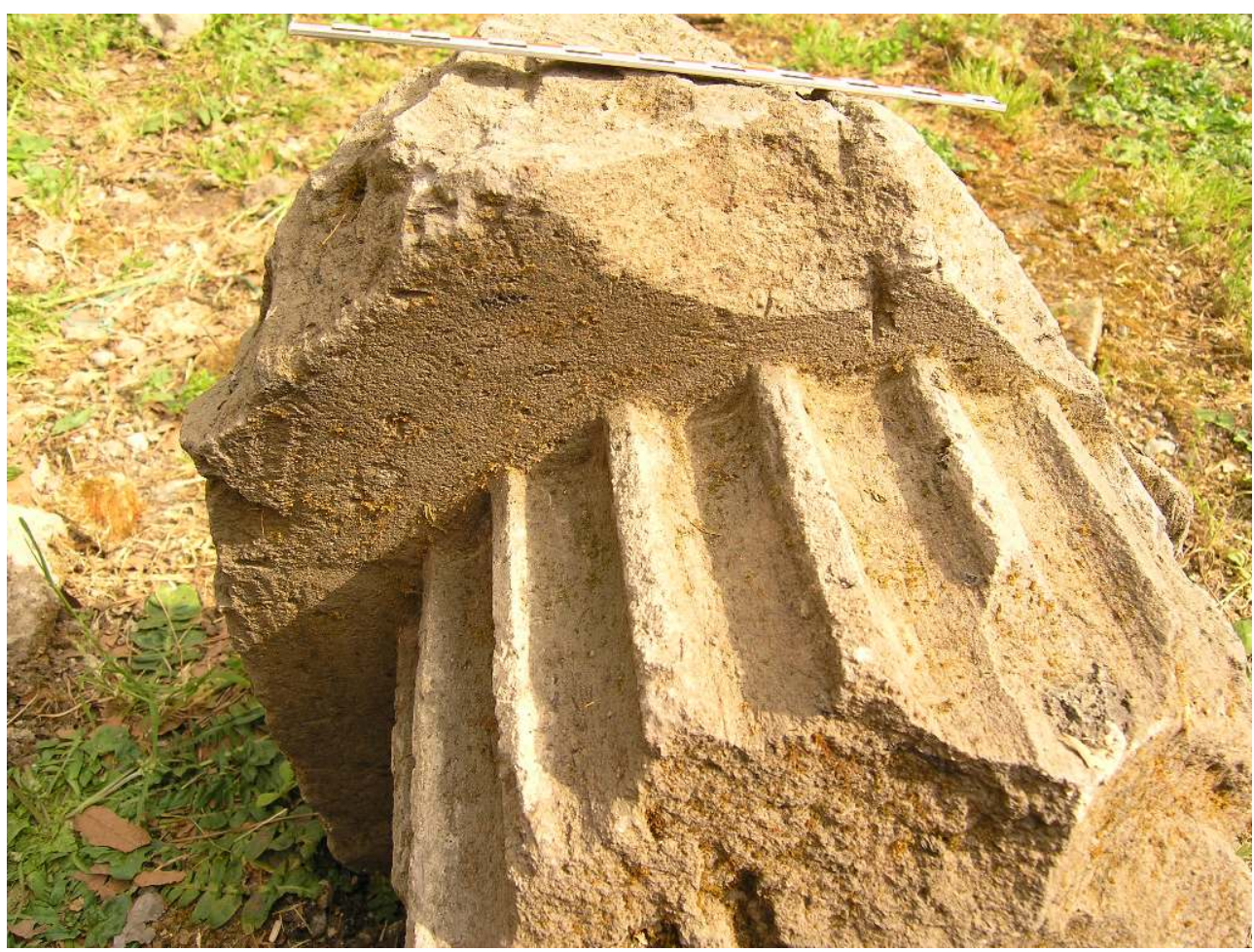

G. Chapelin.

9 Quelques exemplaires présentent aussi un aspect brut, ou tout du moins non finalisé. Il s'agit essentiellement des exemplaires dont le fût n'a pas été cannelé après la pose. Une série en a été remontée sur la via dell'Abbondanza en façade de la Casa dei cenacoli colonnati (IX, 12, 1-2) ${ }^{9}$. Les blocs présentent d'ailleurs encore les traces de tournage à la limite des lits. Ils sont, en outre, voisins à cet endroit d'une série de piliers à demicolonnes engagées quant à eux totalement terminés, abrasés, cannelés et couronnés eux aussi de chapiteaux cubiques (fig. 8). 
Fig. 8 - Vue de détail de la façade à gauche de la Casa dei cenacoli colonnati $(I X, 12,1-2)$ et à droite celle de la Casa del secondo cenacolo colonnato (IX, 12, 3-5) ou deux types d'éléments cohabitent.

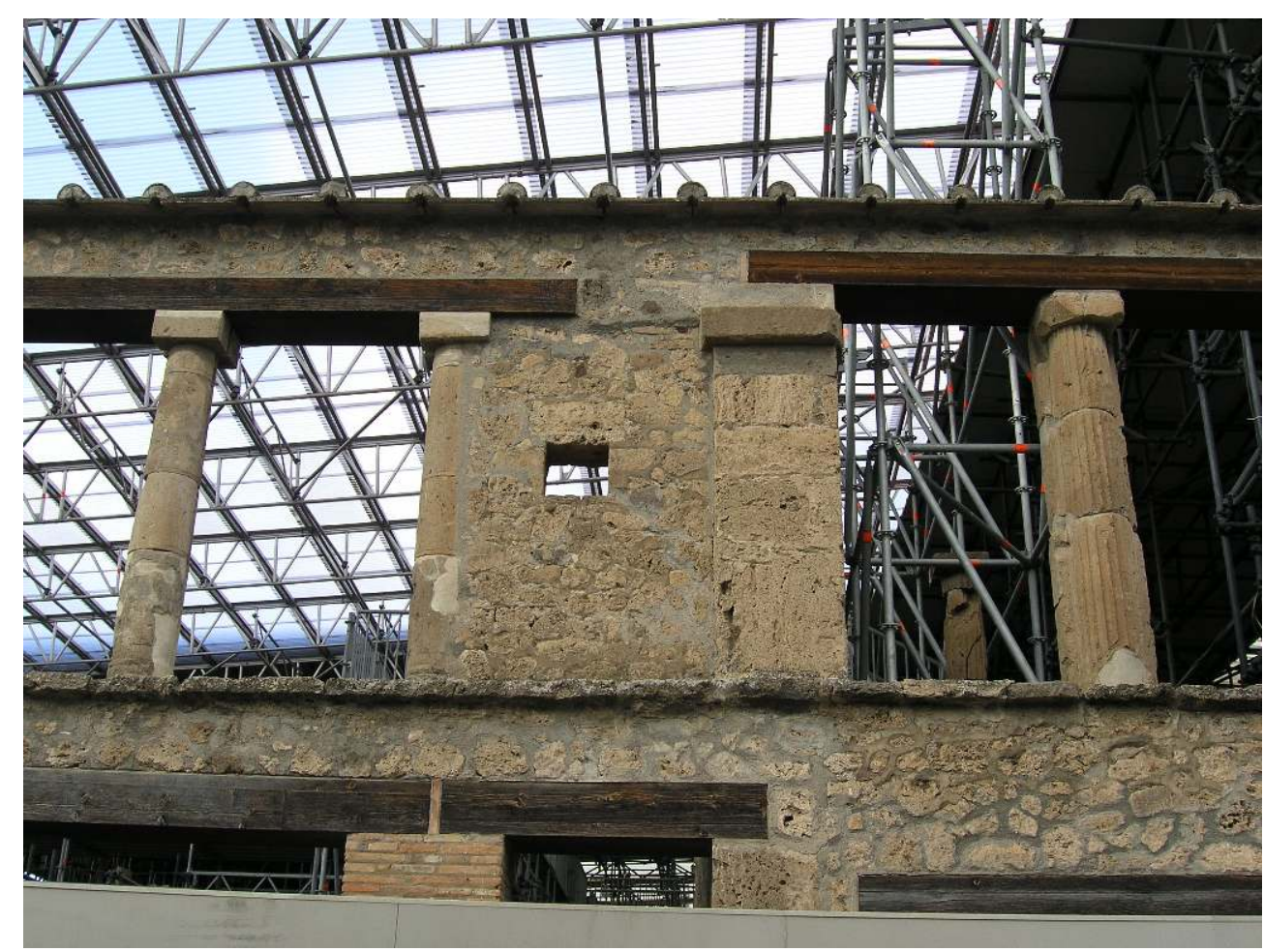

G. Chapelin.

Sur un plan purement technique, chaque catégorie distincte, colonne libre ou pilier à demi-colonne engagée fait l'objet d'une opération de taille spécifique. Si les premiers font systématiquement l'objet d'une approche au tour, ce n'est bien logiquement pas le cas des seconds. Ces modes opératoires ne marquent pas, quoi qu'il en soit, une différence d'atelier mais plutôt une manière spécifique de répondre à des contraintes techniques.

\section{Conclusion et perspectives}

11 Les données collectées dans le cadre de cette recherche depuis 2012 ont permis l'étude du geste et des modes opératoires du tailleur de pierre, tant du point de vue technique que de celui de la stylistique. Un certain nombre de pièces révèle des détails encore peu observés notamment toutes les traces préparatoires qui illustrent les processus de fabrication. Les blocs étudiés jusqu'à présent renvoient pour l'instant l'image de quelques ensembles assez homogènes et peu diversifiés à l'exception de rares blocs qui se distinguent très nettement (un lot de colonnes lisses en calcaire du Sarno et deux lots de colonnes et piliers à demi-colonne engagée de style dorique). Ainsi, il semble, en l'état actuel de nos connaissances, que ces éléments puissent provenir d'officines peu nombreuses, ou tout du moins avec peu de personnel. En revanche, l'idée d'une production régulière déconnectée des périodes d'activités des chantiers, notamment pour les constructions privées, semble difficile à mettre en évidence à partir du simple examen des blocs. Il faudrait en effet, pour pouvoir confirmer cette hypothèse, découvrir un véritable lieu de stockage de pièces non finalisées et prêtes à l'emploi, ou fouiller un niveau de chantier abandonné. 


\section{BIBLIOGRAPHIE}

Bigi 2012 = F. Bigi, I capitelli cubici : una nuova tipologia tutta pompeiana ?, dans Vesuviana : an international Journal of Archaeological and Historical Studies on Pompeii and Herculaneum, 4, 2012, p. 88-110.

De Caro 1985 = S. De Caro, Nuove indagini sulle fortificazioni di Pompei, dans Annali dell'Istituto universitario orientale di Napoli, Sezione di archeologia e storia antica, 7, 1985, p. 75-114.

Kastenmeier et al. 2010 = P. Kastenmeier, G. Di Maio, G. Balassone, M. Boni, M. Joachimski, N. Mondillo, The source of stone building materials from the Pompeii archaeological area and its surroundings, dans Periodico di Mineralogia, 2010, p. 39-58.

Maiuri 1942 = A. Maiuri, L'ultima fase edilizia di Pompei, Rome, 1942 (rééd. 2002).

Maiuri 1958 = A. Maiuri, Portali con capitelli cubici a Pompei, dans Rendiconti dell'Accademia di archeologia, lettere e belle arti di Napoli, 33, 1958, p. 203-218.

\section{NOTES}

1. Le tuf volcanique gris dit «de Nocera » est constitué d'une matrice cendreuse grise qui peut être riche en inclusions de scories noires. Il est à la fois très tendre et léger, donc facile à travailler, et très résistant à la compression ce qui en fait un matériau de construction de choix. Il peut produire, selon les cas, un son très cristallin si on le heurte ne serai-ce qu'avec le doigt. Ce matériau est en fait, d'un point de vue géologique, du faciès gris de l'ignimbrite campanienne issue d'une très grosse éruption des Champs Phlégréens il y a 39000 ans environ et que l'on retrouve dans tout le golfe de Naples (Kastenmeier et al. 2010, p. 40-41).

2. L'agglomération de Pompéi possède évidemment quantité d'autres éléments lapidaires taillés dans d'autres types de matériaux. Pour l'origine des matériaux, voir Kastenmeier et al. 2010 et De Gennaro et al. 2013. Toutefois, le tuf de Nocera garde bien mieux son épiderme que des variétés plus tendres (Pappamonte, calcaire du Sarno, basalte) et est le seul à se prêter à une étude sur les techniques de taille basée sur l'observation des traces techniques. Quant aux variétés plus dures importées (marbres, calcaire blanc dur), elles sont d'emplois plus récent, essentiellement de la période impériale et sortent donc dans l'immédiat du cadre de notre propos. Du reste, elles ne concernent qu'une partie infime du volume de pierres employées sur le site et sont pour la plupart cantonnées à la production d'objets ou rentrent dans la construction de monuments publics.

3. De Caro 1985, p. 107, puis p. 77, qui ne remet pas en cause la datation proposée par A. Maiuri.

4. Maiuri 1942, p. 197.

5. C'est par exemple le cas dans les maisons VII, 9, 47-65 ; VI, 5, 16 et IX, 3, 5.

6. Devant VII, 9, 65 et VI, 4, 10

7. Maiuri 1958 et dernièrement Bigi 2012.

8. Voir aussi à ce propos les entrées de maisons dont les piédroits sont, dans de très nombreux cas, surmontés de chapiteaux cubiques (ainsi les adresses I,1,9 ; VI,7,9 ; VI,9,7 ; VI,10,4 ; VI,10,6 ; VIII,5,28 à titre d'exemple).

9. Un des exemples les plus parfait, qui est d'ailleurs à l'origine des précédentes études sur ces chapiteaux cubiques, est le péristyle de la maison I, 16, 2, dont les tronçons de colonnes et les 
chapiteaux ont été tournés, préparés et jamais finalisés. Seules les bases, attiques, sont totalement abouties. Dans ce cas précis, il s'agit effectivement d'un travail non abouti, probablement pour des raisons financières ou simplement de disponibilité des artisans au moment de la finition.

INDEX

institutions Centre Jean Bérard, Inrap, Soprintendenza speciale per i Beni archeologici di Napoli e Pompei, Ministère des Affaires étrangères (Paris)

Index géographique : Pompéi, Herculanum

Mots-clés : tailleur de pierre, étage, colonnette, pilier à demi-colonne engagée, tuf gris de Nocera, ignimbrite campanienne

\section{AUTEURS}

\section{GUILHEM CHAPELIN}

Centre Jean Bérard (USR 3133 CNRS-EFR) - berard[at]unina.it

\section{GHISLAIN VINCENT}

UMR 5140 Archéologie des Sociétés méditerranéennes, Inrap - ghislain.vincent[at]inrap.fr

\section{MARINA COVOLAN}

Università degli studi di Padova - marina2789[at]hotmail.it 